\title{
Identification of serological biomarkers of infection, disease progression and treatment efficacy for leprosy
}

\author{
John S Spencer ${ }^{1 /+}$, Malcolm S Duthie², Annemieke Geluk ${ }^{3}$, Marivic F Balagon ${ }^{4}$, Hee Jin Kim, \\ William H Wheat', Delphi Chatterjee', Mary Jackson', Wei Li', Jade N Kurihara', \\ Armi Maghanoy ${ }^{4}$, Irene Mallari ${ }^{4}$, Paul Saunderson ${ }^{4}$, Patrick J Brennan', Hazel M Dockrell ${ }^{5}$ \\ ${ }^{1}$ Mycobacteria Research Laboratories, Department of Microbiology, Immunology and Pathology, Colorado State University, \\ Fort Collins, CO, USA ${ }^{2}$ Infectious Disease Research Institute, Seattle, WA, USA ${ }^{3}$ Department of Infectious Diseases, \\ Leiden University Medical Centre, Leiden, The Netherlands ${ }^{4}$ Leonard Wood Memorial Leprosy Research Center, Cebu, Philippines \\ ${ }^{5}$ Department of Immunology and Infection, London School of Hygiene and Tropical Medicine, London, United Kingdom
}

\begin{abstract}
Although leprosy is curable with drug treatment, the identification of biomarkers of infection, disease progression and treatment efficacy would greatly help to reduce the overall prevalence of the disease. Reliable biomarkers would also reduce the incidence of grade-2 disability by ensuring that those who are most at risk are diagnosed and treated early or offered repeated treatments in the case of relapse. In this study, we examined the reactivity of sera from lepromatous and tuberculoid leprosy patients (LPS) against a panel of 12 recombinant Mycobacterium leprae proteins and found that six proteins were strongly recognised by multibacillary (MB) patients, while only three were consistently recognised by paucibacillary patients. To better understand the dynamics of patient antibody responses during and after drug therapy, we measured antibody titres to four recombinant proteins, phenolic glycolipid-I and lipoarabinomannan at baseline and up to two years after diagnosis to investigate the temporal changes in the antibody titres. Reactivity patterns to individual antigens and decreases in antibody titres were patient-specific. Antibody titres to proteins declined more rapidly vs. those to carbohydrate and glycolipid antigens. Compared to baseline values, increases in antibody titres were observed during reactional episodes in one individual. Additionally, antibody responses against a subset of antigens that provided a good prognostic indicator of disease progression were analysed in 51 household contacts of $M B$ index cases for up to two years. Although the majority of these contacts showed no change or exhibited decreases in antibody titres, seven individuals developed higher titres towards one or more of these antigens and one individual with progressively higher titres was diagnosed with borderline lepromatous leprosy 19 months after enrolment. The results of this study indicate that antibody titres to specific M. leprae antigens can be used to monitor treatment efficacy in LPS and assess disease progression in those most at risk for developing this disease.
\end{abstract}

Key words: biomarkers - leprosy - serology - recombinant proteins - household contacts

Leprosy, a chronic mycobacterial infection caused by the obligate intracellular pathogen Mycobacterium leprae, mainly damages the skin and nerves. Patients experience an extraordinary array of cutaneous lesions, peripheral neuropathy and anaesthesia with related disfigurement, deformity and disability along with the social stigma associated with this disease (Scollard et al. 2006). Despite the enormous success of worldwide leprosy control programs since the widespread implementation of multidrug therapy (MDT) in the mid-1980s, 228,474 new cases were recorded in 2010 among the 130 countries reporting, with $9 \%$ of these cases in children and $5.8 \%$ of cases presenting with advanced grade- 2 disability (WHO 2011). Although it is generally accepted that the majority of cases are spread through contact

Financial support: NIH/NIAID (N01 AI-25469, R01 AI-47197, 1 R01 AI-082575), MAGRALEPRE (to AG and JSS), Netherlands Leprosy Relief (ILEP\#:7.01.02.48)/Turing Foundation/IDEAL

+ Corresponding author: john.spencer@colostate.edu

Received 3 June 2012

Accepted 25 July 2012 with those with untreated multibacillary (MB) disease or with individuals with subclinical undiagnosed leprosy, little is known about the true distribution of this disease and what factors are important in the transmission and progression of symptoms in susceptible individuals. It is known is that the rates of $M$. leprae infection are likely many times greater than indicated in newly reported case trends and that leprosy will remain a health problem for the foreseeable future in many parts of the world (Meima et al. 2004, Moet et al. 2008).

An "Enhanced global strategy for further reducing the disease burden due to leprosy (plan period: 20112015)" (WHO 2009) is being implemented by leprosy control programs in countries in which leprosy is endemic. The World Health Organization has set a global targeted reduction of new cases of grade- 2 disability by $\geq 35 \%$ by the end of 2015 compared to baseline levels recorded at the end of 2010. To achieve this goal, the leprosy control programs will need to reduce the delay in disease diagnosis, administer MDT promptly, ensure that patients fully complete their treatment and confirm during follow-up that patients are cured. Together, these actions should impact the transmission of $M$. leprae in the community and decrease the cases of visible disability. However, achieving this goal has been made more diffi- 
cult by the integration of leprosy control into the general health delivery network in many countries and a reduction in the number of trained leprologists and laboratory technicians, which has led to an increase in misdiagnosis and a failure to treat early and appropriately. A definitive diagnosis still relies on clinical observations and timeconsuming invasive tests to confirm the presence of acid-fast bacilli (AFB) in slit skin smears or immunopathological changes in the biopsies of skin lesions. Such services may be lacking in resource-poor settings. The development of a simple serological test that could be easily deployed in the field to identify those individuals at highest risk of succumbing to leprosy would improve early diagnosis and potentially break the lines of transmission to help reduce the rate of grade-2 disability.

We examined the serological responses of 21 untreated MB leprosy patients (LPs) and nine untreated paucibacillary (PB) LPs at diagnosis (baseline) against a panel of 12 recombinant proteins and the native $M$. leprae cytosolic fraction [M. leprae soluble antigen (MLSA)] to assess serological reactivity patterns across the clinical spectrum. Antibody titres in 12 of the MB cases were examined for at least two years after beginning MDT to understand the dynamics of the antibody response to recombinant $M$. leprae proteins, lipoarabinomannan (LAM) and phenolic glycolipid-I (PGL-I) antigens. In addition, 51 contacts of MB index cases were assessed for the same antibody responses against a subset of key antigens to identify any response patterns that can be used to identify those most likely to develop disease symptoms. The antibody titres in household contact (HHC) group were also followed longitudinally for most individuals for at least two years and several HHC developed progressively higher antibody titres during this period. One of these HHC was diagnosed with borderline lepromatous (BL) leprosy at an early stage during routine follow-up after increasing titre results were provided to the clinical staff at the Cebu Skin Clinic. Our results suggest that incorporation of simple and objective serological tests to monitor changes in antibody titres in HHC that have the highest risk of $M$. leprae infection could be an effective strategy to identify patients with early disease progression. Serological tests will lead to more rapid diagnosis and treatment prior to the development of the advanced disease, which will have a profound effect on reducing neurologic impairment and grade-2 disability.

\section{SUBJECTS, MATERIALS AND METHODS}

Subjects and samples - LPs [2 BL (designated MB-3 and MB-7) and 10 lepromatous (LL) patients] were diagnosed at the Cebu Skin Clinic and Leonard Wood Memorial (LWM) Center for Leprosy Research, Cebu, Philippines, based on histological findings and clinical observations determined by experienced leprologists and a leprosy pathologist as previously described (Spencer et al. 2011). Patients were categorised according to the Ridley-Jopling classification (Ridley \& Jopling 1966). Bacterial indices $(\mathrm{BI})$ were determined (a measure of the number of AFB found in the dermis based on a logarithmic scale from 0 at the tuberculoid end of the spectrum to $6+$ at the lepromatous end of the spectrum) and patient sera were collected at the time of diagnosis prior to beginning MDT. Except for one individual who developed dapsone sensitivity, patients were then placed on standard MDT and returned for follow-up bleeds at months 1, 2, 3, 6, 9 and 12 and every three months thereafter until at least two years post-diagnosis. MB patients received WHOrecommended MDT for one year with the following three-drug regimen: $600 \mathrm{mg}$ rifampicin once per month, $300 \mathrm{mg}$ clofazimine during the first month followed by $50 \mathrm{mg}$ daily and $100 \mathrm{mg}$ dapsone daily. The patient who developed dapsone sensitivity received $400 \mathrm{mg}$ of loxacin once per month instead of dapsone. Patients who experienced reactional states (type 1 reversal reaction or type 2 erythema nodosum leprosum) were treated with 20-40 $\mathrm{mg}$ prednisone per day with $5 \mathrm{mg}$ decreases every two weeks once neuritis and lesion swelling subsided. The 20 $\mathrm{mg}$ dose was prescribed for reactions without neuritis, while the $40 \mathrm{mg}$ dose was used for reactions with neuritis. The timing and duration of the reactional episodes were recorded. Additional borderline tuberculoid (BT)/ tuberculoid (TT) $(n=3)$ and BL/LL $(n=9)$ LP sera came from well-characterised samples that had been analysed in a previous study (Spencer et al. 2011).

HHC were defined as individuals who resided with an untreated MB index case for at least six months at the time of diagnosis. Most HHCs were related in some way to the index case (most commonly spouse, parent or sibling). Between one-four HHCs were enrolled for each index case. A total of 51 contacts of $21 \mathrm{MB}$ patients (including the aforementioned $12 \mathrm{MB}$ patients enrolled to assess the effect of MDT on antibody responses) were enrolled after identifying the index case. All HHCs were thoroughly examined and deemed healthy and without any clinical signs or symptoms of the disease at the time of enrolment. A baseline serum sample and follow-up bleeds were obtained every three months for at least two years after enrolment, during which each contact was examined for any signs or symptoms of the disease at follow-up visits. Serum samples from all sources were anonymised and coded to protect donor identities and were obtained with informed consent and/or with permission from the institutional review board of LWM. All procedures were in accordance with the ethical standards of the Helsinki Declaration of 1975 (revised in 1983) regarding human subjects. Sample collection from patients and HHC began in April 2009 and was completed in December 2011. The characteristics of the individuals in the study groups are listed in Table I.

Cloning and purification of recombinant $M$. leprae proteins - DNA sequences encoding 12 full-length $M$. leprae proteins were cloned from genomic DNA as previously described (Spencer et al. 2005, Reece et al. 2006). Purified recombinant proteins were produced by the Infectious Disease Research Institute (IDRI), Seattle, WA or Colorado State University (CSU), Fort Collins, $\mathrm{CO}$, USA. The proteins from IDRI that were selected for investigation included ML0411, ML2055, ML0091, ML0405 and leprosy IDRI diagnostic (LID-1), which is a fusion protein of ML2331 and ML0405. The proteins from CSU that were selected were ML2028, ML2038, ML1795, ML0557, ML0380, ML0286 and ML0050. 
Other antigens - The disaccharide epitope of PGL-I was synthesised and coupled to bovine serum albumin (ND-O-BSA) as previously described (Cho et al. 1984) with modifications (Zhang et al. 2010). The native MLSA was prepared as previously described (Marques et al. 1998). LAM was purified from whole M. leprae cells as previously described (Torrelles et al. 2004). Certain CSU-produced recombinant proteins, native MLSA, ND-O-BSA and LepLAM are available through the Biodefense and Emerging Infections Research Resources Repository (beiresources.org/TBVTRMResearchMaterials/tabid/1431/Default.aspx).

Western blot analysis - Approximately $0.25 \mu \mathrm{g}$ of each recombinant protein was separated using 15\% sodium dodecyl sulfate polyacrylamide gel electrophoresis and transferred to nitrocellulose membranes. Five micrograms of the native MLSA subcellular fraction were loaded in each lane. The membranes were blocked with $1 \%$ BSA in phosphate buffered saline $0.05 \%$ Tween 80 , probed with individual serum samples at a 1:5,000 dilution overnight and developed as previously described (Spencer et al. 2011). The reactivity of each serum sample to each of the recombinant proteins or MLSA was subjectively graded based on the thickness and intensity of the band. Bands given a $3+$ demonstrated the strongest intensity and grades of $2+$ and $1+$ showed progressively less intense staining or thinner bands. A grade of \pm was given to weak, but detectable bands and no visible reaction was scored as (-). Two readers independently scored the bands with few discrepancies $(<5 \%)$, which were generally only one grade higher or lower.

ELISA - The recombinant proteins LID-1 and ML2028 and the other antigens ND-O-BSA and LAM were coated onto high-affinity polystyrene Immulon IV 96-well ELISA plates (Dynex Technologies, Chantilly, VA) at $100 \mathrm{ng}$ per well in $100 \mu \mathrm{L}$ of $0.1 \mathrm{M}$ sodium carbonate/bicarbonate buffer, $\mathrm{pH} 9.6$ at $4^{\circ} \mathrm{C}$ overnight. Serum samples were diluted 1:200 as previously described (Spencer et al. 2011). The absorbance at 405 $\mathrm{nm}$ was obtained using a VersaMax Pro plate reader (Molecular Devices, Sunnyvale, CA) at 15 min after the addition of the substrate.

TABLE I

Sample group characteristics

\begin{tabular}{lcccc}
\hline $\begin{array}{l}\text { Sample } \\
\text { category }\end{array}$ & $\begin{array}{c}\text { Male } \\
(\mathrm{n})\end{array}$ & $\begin{array}{c}\text { Female } \\
\text { (n) }\end{array}$ & $\begin{array}{c}\text { Median age } \\
\text { (range) }\end{array}$ & $\begin{array}{c}\text { BI } \\
\text { (range) }\end{array}$ \\
\hline $\mathrm{MB}^{a}(12)$ & 10 & 2 & $27(18-69)$ & $1.7+-4.7+$ \\
$\mathrm{PB}^{b}(6)$ & 4 & 2 & $39(35-49)$ & 0 \\
$\mathrm{HHC}^{(51)}$ & 23 & 28 & $22(9-62)$ & NA \\
\hline
\end{tabular}

$a$ : 10 lepromatous and two borderline lepromatous, all with $>$ six countable lesions; $b$ : six borderline tuberculoid, all with one or two countable lesions; NA: not available.

\section{RESULTS}

Baseline seroreactivity of LP sera against recombinant proteins and native MLSA - We performed Western blot analysis of $21 \mathrm{BL} / \mathrm{LL}$ and nine BT/TT LP sera samples to examine their reactivity patterns with 12 recombinant proteins and the native M. leprae cytosolic subcellular fraction. The reactivity patterns are shown in Table II and representative blots for three BL/LL and three TT/ BT individuals are shown in Fig. 1. The protein antigens that showed the strongest overall responses in the BL/ LL group were LID-1 (100\%), ML2028 (Ag85B) (100\%), ML2038 (100\%), ML0405 (100\%), ML2055 (95\%) and ML0286 (95\%), with LID-1 and ML2028 (Ag85B) showing the most intense responses of all recombinant proteins tested. Serological reactivity patterns for the remaining proteins were weaker and/or more variable towards ML0411 (90\%), ML0091 (86\%), ML0050 (CFP10) $(86 \%)$, ML0380 (10 kDa GroES) (62\%), ML0557 (29\%) and ML1795 (0.5\%). As expected, the reactivities of the TT/BT sera were much weaker than BL/LL sera, but some of the antigens were well recognised, including ML2028 (100\%), ML0286 (100\%), ML2038 (100\%) and LID-1 (78\%). Whereas nearly all LL patients recognise PGL-I, the strength of the recognition of other protein antigens by TT/BT patients is novel. The native cytosolic subcellular fraction is mainly used to detect the relative intensity of reactivity towards LAM, which is indicated by a smear in the molecular weight range of 20-40 $\mathrm{kDa}$. However, other proteins were also detected, such as ML0380. For patients classified as BL/LL, $62 \%$ recognised ML0380, while none of the BT/TT cases reacted with this protein, which is in accordance with previously reported results (Spencer et al. 2011). Approximately $76 \%$ of the BL/LL patient sera showed very strong reactions to LAM ( $\geq 2+$ intensity), while less than half ( $44 \%)$ of the TT/BT sera demonstrated this intensity. The intensity of this smear in the 20-40 kDa range by immunoblot analysis correlated with the antibody titre to purified M. leprae LAM in the ELISA assay. Collectively, these results showed that each individual generates unique response patterns to various $M$. leprae antigens and that the intensities of the responses to certain antigens are predictive of the clinical disease status.

Longitudinal effect of MDT treatment on antibody titres against $M$. leprae antigens - The antibody titres for all $12 \mathrm{MB}$ index cases were determined by Western blot analysis and ELISA to examine the changes in responses to recombinant protein antigens, PGL-I and LAM over the two-year observation period. The Western blotting results for three of these individuals towards the four recombinant proteins (LID-1, ML2055, ML2028 and ML0050) and native MLSA, which provided the strongest reactions that changed the most over time, are presented in detail in Fig. 2 and the corresponding ELISA results are shown in Fig. 3.

Case MB-1 was initially diagnosed with BL leprosy with a BI of $4.0+$, which was reduced slightly to $3.7+$ after one year of MDT. The treatment course was uneventful with no reactional episodes. The initial titres against all four recombinant proteins and native MLSA were very 
strong and did not appear to significantly decrease during the first six months of therapy. The antibody titres to these proteins did decrease to a greater extent by 24 months, with the reactivity against ML2028 and ML0050 decreasing the most, as shown by immunoblot (Fig. 2A). The decrease in titre towards LID-1 is shown in the ELISA assay by a decrease in optical density (OD) from 1.20 at baseline to 0.77 one year after the end of MDT (Fig. $3 \mathrm{~A})$. However, the antibody titres to PGL-I and LAM in this individual show little, if any, decrease by ELISA.

Case MB-2 was diagnosed with LL disease with a BI of 3.5+, which was reduced to $2.5+$ after completing one year of MDT. This individual had several type
1 reversal reactions at months 1,9 and 12 and received palliative steroid treatment in each instance to dampen the inflammatory response. The intensities of the Western blot bands corresponding to the protein antigens LID-1, ML2055 and ML2028 increased significantly from baseline to one month after beginning MDT, which was likely the result of the reactional episode (Fig. 2B). Reactional episodes are often linked to successful treatment, in which death of the bacilli causes a release of antigens and stimulates cell-mediated and antibody immune responses. After this initial spike in antibody titres to the protein antigens, the antibody response stabilised to a slightly lower level one year after ending MDT.

TABLE II

Western blot reactivity patterns of recombinant Mycobacterium leprae proteins and native M. leprae soluble antigen (MLSA) with 21 boderline lepromatous (BL)/lepromatous (LL), nine borderline tuberculoid (BT)/tuberculoid (TT) and nonendemic control (NEC) serum samples to identify the best antigen candidates for screening by Western blot

\begin{tabular}{|c|c|c|c|c|c|c|c|c|c|c|c|c|c|c|}
\hline BI & ID & 0411 & LID-1 & 2055 & 0286 & 2028 & 0091 & 0405 & 0557 & 2038 & 1795 & 0050 & 0380 & MLSA \\
\hline $6+$ & LL38 & ++ & +++ & ++ & + & ++ & \pm & ++ & - & ++ & - & +++ & ++ & +++ \\
\hline $5+$ & LL12 & + & +++ & ++ & + & +++ & + & ++ & ++ & ++ & \pm & + & +++ & +++ \\
\hline $6+$ & LL31 & ++ & +++ & \pm & + & +++ & \pm & ++ & - & + & - & ++ & - & +++ \\
\hline $5+$ & LL39 & ++ & +++ & ++ & + & +++ & \pm & ++ & - & +++ & - & ++ & + & ++ \\
\hline $6+$ & LL4 & \pm & +++ & ++ & - & ++ & \pm & ++ & - & + & - & \pm & +++ & + \\
\hline $6+$ & LL22 & + & +++ & ++ & + & +++ & + & ++ & - & ++ & - & ++ & ++ & +++ \\
\hline $5+$ & LL27 & \pm & +++ & ++ & + & +++ & + & ++ & + & ++ & - & \pm & ++ & ++ \\
\hline $5+$ & LL19 & ++ & +++ & ++ & + & +++ & + & ++ & - & ++ & - & \pm & ++ & + \\
\hline $5+$ & BL11 & ++ & +++ & ++ & + & +++ & + & ++ & - & ++ & - & +++ & + & ++ \\
\hline $4.0+$ & MB-1 & + & +++ & ++ & \pm & +++ & + & ++ & - & + & - & +++ & - & +++ \\
\hline $3.5+$ & MB-2 & + & +++ & ++ & \pm & + & + & ++ & \pm & + & - & \pm & \pm & + \\
\hline $3.2+$ & MB-3 & ++ & +++ & ++ & \pm & + & ++ & ++ & - & + & - & \pm & - & + \\
\hline $4.33+$ & MB-4 & - & +++ & ++ & \pm & ++ & + & ++ & - & + & - & + & - & ++ \\
\hline $4.7+$ & MB-5 & \pm & ++ & + & \pm & +++ & - & ++ & - & + & - & - & + & ++ \\
\hline $3.8+$ & MB-6 & + & +++ & ++ & + & + & \pm & ++ & \pm & + & - & ++ & - & +++ \\
\hline $1.7+$ & MB-7 & \pm & +++ & - & + & + & - & ++ & - & + & - & \pm & - & +++ \\
\hline $2.83+$ & MB-8 & - & \pm & ++ & + & + & - & \pm & - & + & - & - & - & ++ \\
\hline $4.0+$ & MB-9 & + & +++ & ++ & + & ++ & + & ++ & \pm & + & - & ++ & + & +++ \\
\hline $3.33+$ & MB-10 & \pm & +++ & \pm & \pm & +++ & - & ++ & - & + & - & - & - & +++ \\
\hline $4.33+$ & MB-11 & ++ & +++ & ++ & + & +++ & + & ++ & - & + & - & ++ & + & ++ \\
\hline $4.7+$ & MB-12 & + & +++ & ++ & + & +++ & ++ & ++ & \pm & ++ & - & +++ & +++ & ++ \\
\hline Positive (\%) & & 90 & 100 & 95 & 95 & 100 & 86 & 100 & 29 & 100 & 0.5 & 86 & 62 & 100 \\
\hline 0 & BT53 & \pm & \pm & - & + & + & - & \pm & - & + & - & - & - & +++ \\
\hline 0 & ВT57 & - & \pm & \pm & + & + & - & - & - & + & - & \pm & - & +++ \\
\hline 0 & TT51 & - & \pm & - & + & + & - & \pm & - & + & - & \pm & - & + \\
\hline 0 & PB-1 & - & \pm & - & + & + & - & \pm & - & + & - & \pm & - & +++ \\
\hline 0 & PB-2 & - & + & - & + & + & - & \pm & - & + & - & - & - & - \\
\hline 0 & PB-3 & - & - & - & \pm & + & - & - & - & + & - & - & - & + \\
\hline 0 & PB-4 & - & - & \pm & \pm & + & - & - & + & + & - & - & - & + \\
\hline 0 & PB-5 & - & \pm & - & + & ++ & - & - & - & + & - & \pm & - & ++ \\
\hline 0 & PB-6 & - & \pm & \pm & ++ & + & - & - & - & + & - & - & - & + \\
\hline Positive (\%) & & 11 & 78 & 33 & 100 & 100 & 0 & 44 & 11 & 100 & 0 & 44 & 0 & 89 \\
\hline NA & NEC14 & - & - & - & - & - & - & - & - & - & - & - & - & - \\
\hline
\end{tabular}


However, the responses to PGL-I and particularly LAM showed unexpected increases by Western blotting (Fig. 2B, panel at 24 months) and ELISA (Fig. 3B).

Case MB-3 was initially diagnosed with BL disease with a BI of 3.2+, which was reduced by almost two logs to $1.33+$ after one year of MDT. This patient experienced neuritis during early MDT and was treated with low dose steroids, which were tapered off by six months, without the occurrence of an explicit reactional episode. The patient's titres against the protein antigens LID-1 and ML2055 were particularly strong at baseline, but showed markedly decreased intensities by six months after beginning MDT and were barely detectable at 24 months (Fig. 2C). The response to ML0050 was detectable before treatment initiation, but disappeared by month 3. During this same time period, this patient experienced clearance of skin lesions after only a few months of treatment.

ELISA results showing the antibody titres against LID-1, PGL-I (ND-O-BSA) and LAM over the two-year period for these same three patients are shown in Fig. 3. Patient MB-1 had a slight decrease in the titres against PGL-I and LAM ( $8 \%$ and $4 \%$, respectively), whereas there was a more marked decline in the titre against LID-1 with a baseline OD of 1.20 that decreased by $36 \%$ to 0.77 at the end of two years. Patient MB-2 showed a steady decrease in the response to LID-1 from OD 0.95-0.51, but this patient's anti-PGL-I titre was stable from six-24 months. The anti-LAM titre increased during the last 12 months of observation from OD 1.57-1.90. For patient MB-3, there were marked decreases in the titres against all three antigens, particularly during the first three months of drug therapy. The titres against PGL-I and LAM decreased by
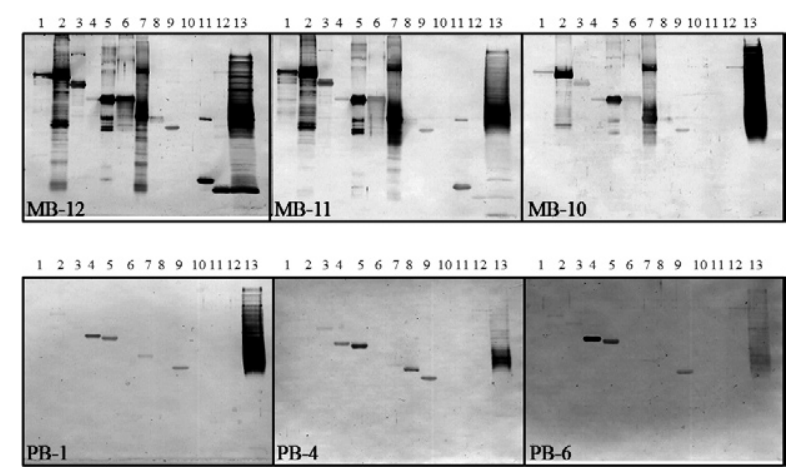

Fig. 1: Western blots of 12 recombinant proteins and the native $M y$ cobacterium leprae cytosolic protein probed with three representative lepromatous [multibacillary (MB)-12, MB-11 and MB-10] and three tuberculoid [paucibacillary (PB)-1, PB-4 and PB-6] leprosy patient serum samples. Approximately $0.25 \mu \mathrm{g}$ of each recombinant protein and $2.5 \mu \mathrm{g}$ of M. leprae soluble antigen (MLSA) were run in individual Lanes: 1: ML0411, $45 \mathrm{kDa}$ serine rich antigen; 2: leprosy IDRI diagnostic (LID-1) fusion protein; 3: ML2055, 29.6 kDa modD; 4: ML0286, $36.7 \mathrm{kDa}$ fructose bisphosphate aldolase; 5: ML2028, $34.8 \mathrm{kDa}$ Ag85B; 6: ML0091, $28 \mathrm{kDa}$ antigen precursor; 7: ML0405, $23.8 \mathrm{kDa}$ conserved hypothetical protein (part of LID-1); 8: ML0557, $24.9 \mathrm{kDa}$ lprG lipoprotein; 9: ML2038, 18.3 kDa bacterioferritin; 10: ML1795, $18 \mathrm{kDa}$ heat shock protein; 11: ML0050, $10.9 \mathrm{kDa}$ CFP-10; 12: ML0380, 10.8 kDa GroES chaperonin; 13: native MLSA. Blots were probed with a 1:5,000 dilution of patient serum.
$46 \%$ and $51 \%$, respectively, while that against LID-I declined by $65 \%$ over the 24 -month period.

Overall, these results indicate that the antibody response to protein antigens declines more rapidly over time, while the responses against carbohydrate and glycolipid antigens tend to decay more slowly or even remain elevated, particularly in individuals with a very high initial bacillary load.

Assessment of antibody responses in 51 healthy HHC over a two-year period - Healthy HHC were enrolled after each index case was diagnosed and a baseline bleed was obtained. The reactivities of the antibodies in the baseline samples against three key antigens, including LID-1 (which had been shown in a previous study to elicit a positive antibody response in individuals who were diagnosed with clinical leprosy up to one year prior to the onset of disease symptoms) (Duthie et al. 2007), ML2028 (Ag85B) and native MLSA, which was examined to assess reactivity against LAM, were determined by Western blot analysis, as shown in Fig. 4. Responses against the protein antigens LID-1 and ML2028 were much weaker than those observed in the MB index cases (index case MB-12 is included in Fig. 4 as an example), although the HHCs frequently demonstrated strong titres against LAM. The initial assessment of baseline reactivity patterns by Western blot analysis showed responses of $\geq 1$ against LID-1 (8/51 HHCs, 16\%), ML2028 (23/51, $45 \%)$ and MLSA (14/51, 27\%). Two of these individuals, HHC 12-2 and HHC 18-2, showed much stronger reactivity (2+) towards both LID-1 and ML2028 compared to other HHC, whereas these subjects demonstrated only weak reactivity with MLSA. The antibody titres against LAM and PGL-I were determined by ELISA for 48 of these HHC (3 individuals did not have complete followup serum samples taken) for up to eight serial bleeds over the 24-month period. No change or slight decreases in the titres against LAM or PGL-I were observed in $41 / 48(85 \%)$ of these individuals, while seven HHC showed some increase in their response against one of these antigens. The antigen reactivity patterns of these seven HHC were re-evaluated to identify changes in reactivity against LID-I, ML2028 and MLSA between baseline and later samplings. Two of these individuals showed increased reactivities against the protein antigens PGL-I and LAM. HHC 5-1 had a very strong titre against LAM by immunoblot at baseline and this titre increased somewhat by ELISA and immunoblot at 21 months. More importantly, this patient demonstrated reactivity against the $10 \mathrm{kDa}$ GroES protein (ML0380) in MLSA at baseline and this titre increased considerably at 21 months. Antibodies to this protein are found in $>$ $50 \%$ of MB patients, but is negative in all PB individuals we have examined to date. Thus, a positive response to ML0380 in a HHC would likely place this individual in a higher risk category and necessitate close monitoring for signs of the disease. The second subject with increased reactivities described above, HHC 18-1, showed a substantial increase in the anti-LAM titre at 15 months with a corresponding increase in reactivity against LID-1 by immunoblot. At the time of submission, the disease status of these individuals remained unchanged. 
Further analysis of HHC 12-2 and HHC 18-2 over a two-year period - Because antibody titres to LID-1 have previously been shown to increase prior to clinical diagnosis (Duthie et al. 2007) we were especially interested in those HHCs who showed stronger reactivities against this antigen. HHC 12-2 and HHC 18-2 showed the strongest responses against LID-1 and ML2028 at baseline. Analysis of serial serum samples from HHC 18-2 showed no change in the strength of the response to LID-1 or ML2028, but showed a decrease in the response to LAM (data not shown). In the case of HHC 12-2, Western blot analysis of serial bleeds taken over the course of 15 months showed no change in the strength of the response to LID-1 or ML2028, but there was an increase in the antibody titre against MLSA (specifically against the 20-40 kDa region associated with LAM reactivity) at six months. This response grew progressively stronger with each three-month interval (Fig. 5A). Further analysis of the serial serum samples by ELISA confirmed that while the OD corresponding to the response against ML2028 remained essentially unchanged, the results measuring the responses against both anti-PGL-I and anti-LAM showed a progressive increase from the baseline OD of approximately 0.30 to a final $\mathrm{OD}$ of greater than 1.60 (Fig. 5B). The increasing titre in this individual was reported to the director of the clinic (MFB) in April 2011. Two months later, this individual was confirmed to have BL leprosy with a bacillary load of $1.7+$ on a routine follow-up visit. Because this diagnosis was made early when the bacillary load was still relatively low and MDT was immediately initiated, it is likely that further damage or neurological impairment was prevented.

Evidence that treatment of the index case influences antibody titres in HHCs - In addition to examining all serial bleeds for changes in antibody titres to PGL-I and LAM by ELISA, the baseline and final serum samples from each HHC were probed against LID-1, ML2028 and
MLSA by Western blot analysis to identify any changes in antibody titres in HHCs once the index case in the household completed MDT. In 17/51 HHCs (33\%), there was a detectable decrease in the reactivity against one or more antigens. The results obtained from four HHCs (Fig. 6) showed marked decreases in reactivity against ML2028 and two individuals (HHC6-2 and HHC12-3) showed decreased reactivities towards LAM. Notably, HHC12-1 and HHC12-3 showed decreased responses to these antigens, whereas HHC12-2, a sibling of index case MB-12, was the only individual who developed a rapidly rising titre and progressed to clinical disease.

\section{DISCUSSION}

Clinical examination and bacteriological and histological findings continue to be the methods of choice for the diagnosis and follow-up care of LPs. The availability of better tools for clinicians and health care workers to assign the proper treatment regimen, monitor the efficacy of therapy, detect disease relapse and identify biomarkers of infection and disease progression in individuals most at risk for developing this disease, particularly for contacts of MB index cases, is sorely needed. The presence of IgM antibodies to the M. leprae-specific PGL-I has been used in the past to classify patients for treatment purposes (Bührer-Sékula et al. 2003) and the magnitude of the antibody titre correlates strongly with the bacillary load. However, detection of the anti-PGL-I antibody in those at the tuberculoid end of the spectrum (TT/BT) is low, usually approximately $20-40 \%$. Recent studies have shown that responses to protein antigens can be used to characterise this disease. A number of these antigens, including ML2028, ML2038 (Spencer et al. 2011) and the fusion protein LID-1 (Duthie et al. 2010, 2011) have been shown to induce very strong antibody responses in virtually all BL/LL cases. A previous study in which HHCs of MB patients were bled four years prior to clinical diagnosis showed that seven/11 (64\%) developed antibody re-

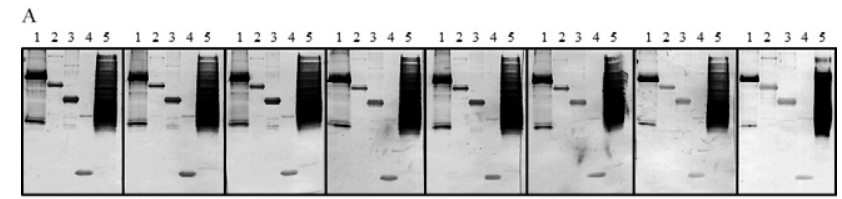

B

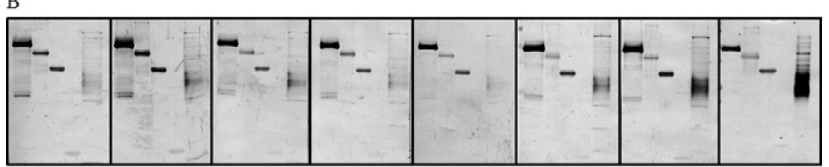

$\mathrm{C}$

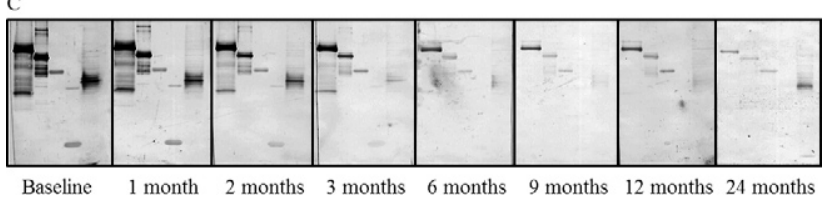

Fig. 2: Western blot of four recombinant proteins and Mycobacterium leprae cytosolic protein, M. leprae soluble antigen (MLSA), probed with serial serum samples from three index cases at baseline and up to 24 months after multidrug therapy. Lane 1: leprosy IDRI diagnostic; 2: ML2055; 3: ML2028; 4: ML0050; 5: native MLSA; A: multibacillary (MB)-1, initial bacterial index (BI) of 4.0+; B: MB-2, initial BI of 3.5+, with reversal reactions at months 1,9 and 12 ; $\mathrm{C}$ : MB-3, initial $\mathrm{BI}$ of $3.2+$. 
sponses to LID-1 up to one year prior to the development of clinical symptoms. These antibody titres were higher and occurred much sooner than the increases in the antiPGL-I IgM responses observed in the same individuals (Duthie et al. 2007). It was also shown that a heightened IgG titre against LID-1 was a good indicator of early infection and active disease, as titres remained low or undetectable in contacts who did not progress to disease. Our study indicates that a strong response to LID-1 and ML2028 may represent a biomarker of disease progression because a contact that developed leprosy (HHC12-2) showed the strongest response to both of these antigens 19 months before clinical diagnosis. Nevertheless, the antibody titres against LID-1 and ML2028 remained static from baseline through 15 months, whereas those against PGL-I and LAM increased significantly starting at six months. The increased titres against these protein antigens are likely dependent on the bacterial load, as increased band intensity by Western blot analysis or significant elevation in the ELISA signal against these two antigens occurred in the individuals with BIs greater than 3.0. However, there was significant variability in these responses among MB cases. MB-7, who had a BI of only $1.7+$, had a very strong $3+$ reaction to LID-1 compared to MB- 8 , who had a BI of $2.83+$ and whose reactivity to LID-1 was weakly positive $( \pm)$.
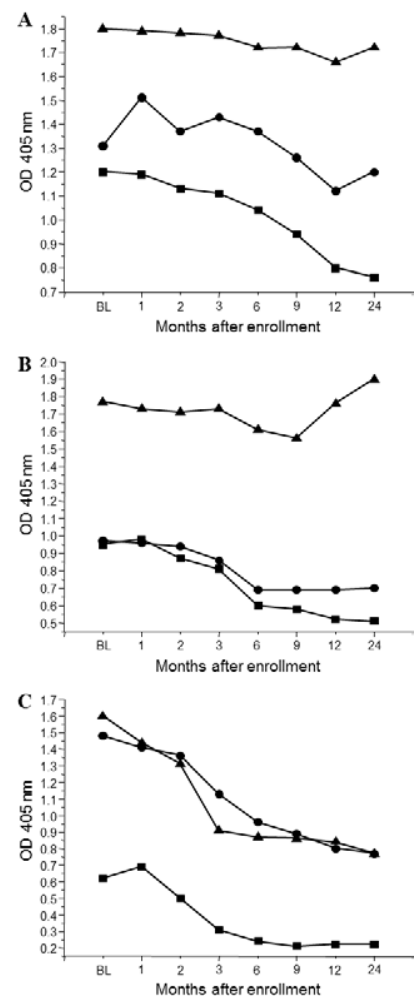

Fig. 3: ELISA assay of serial serum samples from three index cases at baseline and up to 24 months after beginning multidrug therapy to show the effect of treatment on antibody titre to leprosy IDRI diagnostic (LID-1) (closed square), ND-O-BSA (phenolic glycolipid-I) (closed circle), and LepLAM (closed triangle). A: patient multibacillary (MB)-1; B: patient MB-2; C: patient MB-3. OD: optical density.
A number of studies have shown that family members living in close contact with an untreated MB index case have the highest risk for developing this disease (Fine et al. 1997, Cunanan et al. 1998, van Beers et al. 1999). In a large study involving 1,396 HHCs of index cases (79\% of the index cases were MB) in Uberlândia, Brazil over a five-year period, 28 individuals were eventually diagnosed with leprosy, with $75 \%$ of those cases occurring within the first year of enrolment (Goulart et al. 2008). Thus, the percentage of HHCs who eventually developed the disease was $2 \%$, as was the case in our study to date. All HHCs were assessed for the absence or presence of a BCG scar, Mitsuda skin reaction by lepromin injection and antibody titre to PGL-I. It was determined that the individuals at highest risk, with a combined risk factor of 24.5, were those who showed positive PGL-I antibody responses and were negative for a BCG scar and Mitsuda reaction. However, demonstrating a positive anti-PGL-I response indicated a relative risk that was approximately six-fold higher for the occurrence of the disease. Additional risk factors for leprosy in HHCs have been identified in a large prospective cohort study of 21,870 contacts of newly diagnosed LPs in northwest Bangladesh called the COLEP study (Moet et al. 2008). The risk factors identified in this study include contact with cases of PB leprosy with two-five lesions or MB disease, physical closeness, such as residing within the
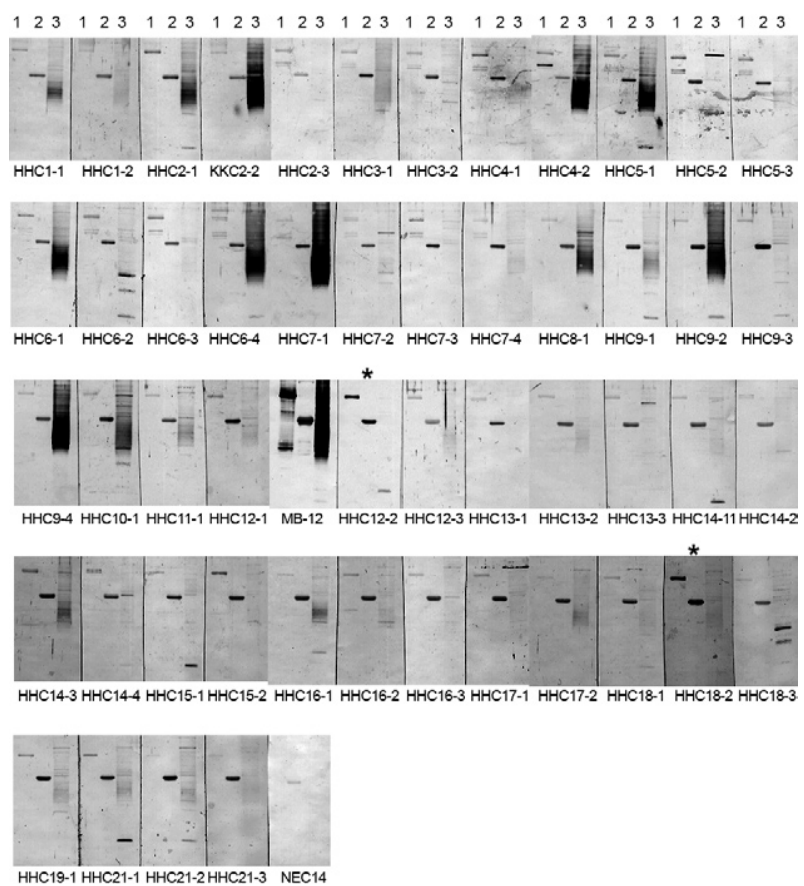

Fig. 4: baseline Western blot reactivity patterns of 51 healthy household contact (HHC) of multibacillary (MB) leprosy patients against leprosy IDRI diagnostic (LID-1) (Lane 1), ML2028 (Lane 2) and native Mycobacterium leprae soluble antigen (Lane 3). Contacts having strong reactivity against both LID-1 and ML2028 (HHC12-2 and HHC18-2) are labelled with an asterisk. Nonendemic control (NEC)14 is a representative NEC healthy serum and MB-12 is the baseline reactivity of a representative index case. 
same household, age, with the highest risk associated with those 15-20 years of age and those older than age 30 , thereby representing a bimodal distribution and genetic relation, as in the case of parents and their children or consanguineous siblings living together within the same household (Moet et al. 2006).

In our study, index case MB-12 had a very high BI at diagnosis $(4.7+)$ with over 20 countable lesions. This patient was diagnosed in late 2009 , but was thought to have had clinical disease for up to five years previously and lived in a household with three blood-related HHCs
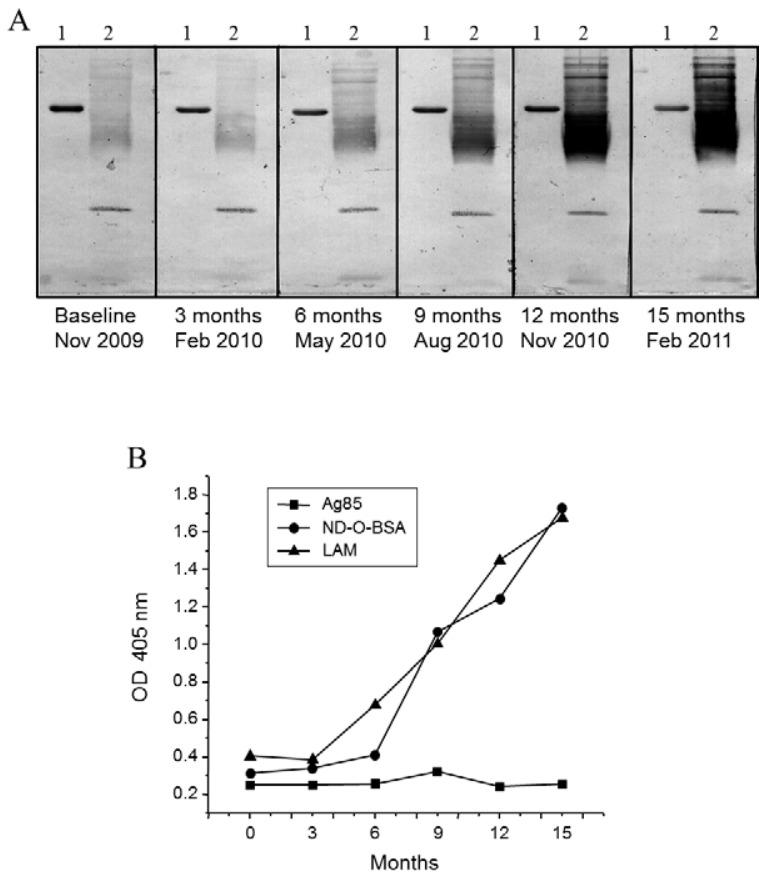

Fig. 5: Western blot and ELISA assay results of serial bleeds of household contact 12-2 showing increase in antibody titre to lipoarabinomannan (LAM) and phenolic glycolipid-I. A: Western blot of recombinant protein ML2028 (Lane 1) and Mycobacterium leprae soluble antigen (Lane 2) with serum samples from baseline through 15 months after enrolment. The reactivity with leprosy IDRI diagnostic was the same intensity as ML2028 and also did not change over time (data not shown); B: ELISA assay of the same serum samples showing the optical density (OD) against ML2028 (Ag85), ND-O-BSA, and M. leprae LAM. for over 12 years. Despite these facts, it was not until 19 months after enrolment that $\mathrm{HHC12}-2$ showed clinical disease signs and was diagnosed. The PGL-I and LAM antibody titres had substantially increased for more than one year prior to diagnosis. Interestingly, the other two family members in this household, HHC12-1 and HHC12-3, showed decreasing titres against both antigens by ELISA and Western blotting. One likely explanation for this decrease is that the main source of $M$. leprae exposure in the household was essentially removed soon after the index case began MDT. It is thought that constant shedding of viable M. leprae bacilli from the nasal secretions of untreated LL cases, estimated to be on the order of up to $10^{7}$ bacilli per day (Davey \& Rees 1974), would result in the continuous exposure and/or re-infection of contacts residing in the household. Repeated exposure to high bacillary loads causes an increase in both cell-mediated immunity and antibody titres to M. leprae antigens (Martins et al. 2012, Geluk et al. 2012). However, treatment of the index case would cause a rapid cessation of shedding of viable bacilli, eventually eliminating exposure to mycobacterial antigens within the household. In the absence of this antigenic stimulus, one would expect the antibody titre to wane. Thus, effective treatment of the index case can have a spillover effect on the antibody responses of those closest to this individual, resulting in a measurable decrease in the antibody titres in HHCs over time. The fact that a measurable decrease in antibody titre to one or more antigens was detected in one third of all HHCs could be interpreted as a clear indication of the impact of the successful treatment of index cases.

Although there was a rapid rise in the antibody titres against PGL-I and LAM beginning at six months in HHC12-2, the antibody responses to LID-1 and ML2028 were unchanged for 15 months as detected by Western blotting and ELISA. When one compares these values with the antibody responses in the index case MB-12, it might be predicted that the very high IgG titres against LID-1 and ML2028 in individuals with very high BIs likely evolved over a long period of time, perhaps over the course of several years. During disease progression, the point at which the bacillary load may exceed a certain tipping point to cause a similar increase in the $\mathrm{IgG}$ titres against protein antigens remains unclear. At the time of diagnosis, the $\mathrm{BI}$ of $\mathrm{HHC12}-2$ was only $1.7+$, whereas the BI for index case MB-12 was 4.7+, indicating a 1,000-fold higher bacillary load. One can calculate what this bacte-

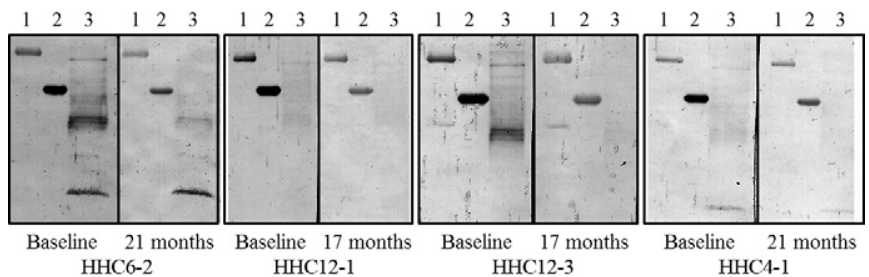

Fig. 6: Western blot of four household contact (HHC) (HHC6-2, HHC12-1, HHC12-3 and HHC4-1) against leprosy IDRI diagnostic (LID-1), ML2028 and Mycobacterium leprae soluble antigen (MLSA) at the beginning of enrolment (baseline) and towards the end of the study showing a decrease in reactivity towards one or more antigens over time. Lane 1: LID-1; 2: ML2028; 3: MLSA. 
rial burden represents by assuming that a $\mathrm{BI}$ of 1.0 represents approximately $10^{4}$ bacilli per gram of tissue (J Barreto, unpublished observations), therefore assuming that a BI of 4.7 would be 1,700 times more than a BI of 1.0. Assuming that $2.0 \times 10^{9}$ bacilli weigh $1 \mathrm{mg}$ and that skin represents $20 \%$ of body weight, an individual weighing $70 \mathrm{~kg}$ with a BI of $4.7+$ would be carrying roughly 0.5 $\mathrm{kg}$ of $M$. leprae throughout his body. It is not surprising that this staggering amount of bacilli causes the extremely high antibody responses to protein, carbohydrate and glycolipid antigens in individuals with very high BIs.

The presence of IgM serum antibodies to PGL-I in LPs has been shown to have potential for classifying patients for treatment purposes, case monitoring, identification of the risk of relapse and identification of HHCs with a higher risk of developing the disease (Oskam et al. 2003, Bührer-Sékula et al. 2003, de Moura et al. 2008). However, the vast majority of PB leprosy cases do not develop detectable antibodies to PGL-I and in areas where leprosy is hyperendemic, as in the Brazilian Amazon in northeastern Brazil, greater than $50 \%$ of young schoolchildren surveyed in some provinces had positive anti-PGL-I responses (Barreto et al. 2011, Salgado et al. 2012). A number of recent reports have shown that $\mathrm{IgG}$ responses to protein antigens could be used as supplemental tools in the development of serological tests to diagnose leprosy, monitor treatment and detect relapse after treatment (Duthie et al. 2011, Geluk et al. 2011). The titres of $\operatorname{IgG}$ antibodies to protein antigens generally correlate with the bacterial load and strong responses in MB patients can rival anti-PGL-I responses. The best protein candidates to assess the IgG response include ML0405, ML2331, ML2055 (Reece et al. 2006, Duthie et al. 2007), ML2028 (Launois et al. 1994, Spencer et al. 2011) and ML2038 (Maeda et al. 2007, Kai et al. 2008, Spencer et al. 2011). A bioinformatics analysis predicted ML0405 to be one of the most promiscuous M. leprae proteins studied to date and to contain $\mathrm{T}$ cell epitopes predicted to be recognised by 50 out of $51 \mathrm{hu}-$ man leukocyte antigen-DR alleles along with seven potential $\mathrm{T}$ cell epitopes and 39 potential B cell epitopes, whereas ML2331 contained 24 potential B cell epitopes (Sampaio et al. 2011). The engineering of these two proteins into the fusion protein LID-1 has been shown to be effective in detecting antibody responses in virtually all MB patients, with specificity and sensitivity of $87 \%$ (Duthie et al. 2007). IgG antibody responses to LID-1 were detected a year in advance of disease symptoms in seven/11 HHCs of MB patients (64\%) who were eventually diagnosed with leprosy. These responses were strikingly more elevated and occurred much earlier than the increases in the anti-PGL-I IgM responses in the same individuals (Duthie et al. 2007). Nevertheless, the responses to LID-1 in PB individuals were comparatively weak. Therefore, work to identify other proteins that are better recognised at the tuberculoid end of the spectrum is on-going. In this study, we confirmed that ML2028 and ML2038 were recognised by almost all PB patient sera. In addition, one of the new antigens tested, ML0286, was similarly recognised by all MB and PB patient sera. This protein, a 1,6-fructose bisphosphate aldolase (Rv0363c, fba ortholog in Mycobacterium tu- berculosis), was recently shown to be important in the glycolytic/gluconeogenic pathway in $M$. tuberculosis, is produced under various axenic growth conditions, including oxygen deprivation, and was found to be expressed in the necrotic core of primary lung granulomas in infected guinea pigs. Thus, this protein may play an important role in nonreplicating bacilli during latent infection (de la Paz Santangelo et al. 2011). Interestingly, ML0286 was also detected in subcellular fractions of armadillo-derived $M$. leprae grown in vivo and had previously been identified by proteomic analysis of the $M$. leprae cytosolic protein fraction (Marques et al. 2004). It may be possible to engineer epitopes from these three proteins (ML2028, ML2038 and ML0286) into a fusion protein that is well recognised by $\mathrm{PB}$ patients in the same way that LID-1 was engineered.

The results of our study indicate that the detection of antibody responses to $M$. leprae protein antigens provides an added benefit to monitoring anti-PGL-I responses alone in HHCs who are most at risk of developing leprosy. Although sensitive, the use of Western blotting as a screening tool to assess antibody titres in HHCs is not practical. Alternatively, an ELISA for the high-throughput analysis of large numbers of samples that incorporates antigens (perhaps as a fusion protein incorporating multiple protein epitopes) recognised by $\mathrm{MB}$ and PB individuals is still in the developmental stage. The development of a rapid protein-based serological assay could be used in nonspecialised settings, such as field conditions, especially in areas where leprosy is still hyperendemic, to more rapidly identify early signs of this disease. Because the majority of HHCs develop a protective immune response and do not progress to disease, a large-scale prospective longitudinal study in HHCs and healthy controls from a hyperendemic region would be critical to determine whether the biomarkers identified in this study can be used as additional tools to more readily predict disease at an early stage as we have shown here, allowing for treatment that would effectively lower disease prevalence and reduce grade-2 disability.

\section{ACKNOWLEDGEMENTS}

To Linda Oskam, for coordinating the activities of these studies, to members of the clinical and technical staff, to Guillerma Lim and Esterlina Tan, for their long service to LWM, and all of the patients who participated at Leonard Wood Memorial and the Cebu Skin Clinic in Cebu.

\section{REFERENCES}

Barreto JG, Guimarães L de S, Leão MR, Ferreira DV, Lima RA, Salgado CG 2011. Anti-PGL-I seroepidemiology in leprosy cases: household contacts and school children from a hyperendemic municipality of the Brazilian Amazon. Lepr Rev 82: 358-370.

Bührer-Sékula S, Smits HL, Gussenhoven GC, van Leeuwen J, Amador S, Fujiwara T, Klatser PR, Oskam L 2003. Simple and fast lateral flow test for classification of leprosy patients and identification of contacts with high risk of developing leprosy. J Clin Microbiol 41: 1991-1995.

Cho S-N, Fujiwara T, Hunter SW, Rea TH, Gelber RH, Brennan PJ 1984. Use of an artificial antigen containing the 3,6-di-O-methy$\beta$-D-glucopyranosyl epitope for the serodiagnosis of leprosy. J Inf Dis 150: 311-322. 
Cunanan Jr A, Chan GP, Douglas JT 1998. Risk of development of leprosy among Culion contacts. Int J Lepr Other Mycobact Dis 66: S78A.

Davey TF, Rees RJ 1974. The nasal discharge in leprosy: clinical and bacteriological aspects. Lepr Rev 45: 121-134.

de la Paz Santangelo M, Gest PM, Guerin ME, Coinçon M, Pham H, Ryan G, Puckett SE, Spencer JS, Gonzalez-Juarrero M, Daher R, Lenaerts AJ, Schnappinger D, Therisod M, Ehrt S, Sygusch J, Jackson M 2011. Glycolytic and non-glycolytic functions of Mycobacterium tuberculosis fructose-1,6-bisphophate aldolase, an essential enzyme produced by replicating and non-replicating bacilli. J Biol Chem 286: 40219-40231.

de Moura RS, Calado KL, Oliveira MLW, Bührer-Sékula S 2008. Leprosy serology using PGL-I: a systematic review. Rev Soc Bras Med Trop 41 (Suppl. II): 11-18.

Duthie MS, Goto W, Ireton GC, Reece ST, Cardoso LPV, Martelli CMT, Stefani MMA, Nakatani M, de Jesus RC, Netto EM, Balagon MVF, Tan E, Gelber RH, Maeda Y, Makino M, Hoft D, Reed SG 2007. Use of protein antigens for early serological diagnosis of leprosy. Clin Vacc Immunol 14: 1400-1408.

Duthie MS, Hay MN, Morales CZ, Carter L, Mohamath R, Ito L, Oyafuso LK, Manini MI, Balagon MV, Tan EV, Saunderson PR, Reed SG, Carter D 2010. Rational design and evaluation of a multiepitope chimeric fusion protein with the potential for leprosy diagnosis. Clin Vacc Immunol 17: 298-303.

Duthie MS, Hay MN, Rada EM, Convit J, Ito L, Oyafuso LK, Manini MI, Goulart IM, Lobato J, Goulart LR, Carter D, Reed SG 2011. Specific IgG antibody responses may be used to monitor leprosy treatment efficacy and as recurrence prognostic markers. Eur $J$ Clin Microbiol Infect Dis 30: 1257-1265.

Fine PE, Sterne JA, Ponnighaus JM, Bliss L, Saul J, Chihana A, Munthali M, Warndorff DK 1997. Household and dwelling contact as risk factors for leprosy in northern Malawi. Am J Epidemiol 146: 91-102.

Geluk A, Bobosha K, van der Ploeg-van Schip JJ, Banu S, Spencer JS, Martins MB, Cho S-N, Franken KLMC, Kim HJ, Bekele Y, Uddin MKM, Hadi SA, Aseffa A, Pessolani MCV, Pereira GMB, Dockrell HM, Ottenhoff THM 2012. New biomarkers for Mycobacterium leprae infection applicable in areas highly endemic for leprosy. J Immunol 188: 4782-4791.

Geluk A, Duthie MS, Spencer JS 2011. Postgenomic Mycobacterium leprae antigens for cellular and serological diagnosis of M. leprae exposure, infection and leprosy disease. Lepr Rev 82: 1-20.

Goulart IMB, Bernardes Souza DO, Marques CR, Pimenta VL, Gonçalves MA, Goulart LR 2008. Risk and protective factors for leprosy development determined by epidemiological surveillance of household contacts. Clin Vacc Immunol 15: 101-105.

Kai M, Nguyen Phuc NH, Hoang Thi TH, Nguyen AH, Fukutomi Y, Maeda Y, Miyamoto Y, Mukai T, Fujiwara T, Nguyen TT, Makino M 2008. Serological diagnosis of leprosy in patients in Vietnam by enzyme-linked immunosorbent assay with Mycobacterium leprae-derived major membrane protein II. Clin Vacc Immunol 15: 1755-1759.

Launois P, N'Diaye NM, Drowart A, Van Vooren JP, Sarthou JL, Lalu T, Millan J, Huygen K 1994. IgG response to purified 65 and 70$\mathrm{kDa}$ mycobacterial heat shock proteins and to antigen 85 in leprosy. Int J Lepr Other Mycobact Dis 62: 48-54.

Maeda Y, Mukai T, Kai M, Fukutomi Y, Nomaguchi H, Abe C, Kobayashi K, Kitada S, Maekura R, Yano I, Ishii N, Mori T, Makino M 2007. Evaluation of major membrane protein-II as a tool for serodiagnosis of leprosy. FEMS Microbiol Lett 272: 202-205.
Marques MAM, Chitale S, Brennan PJ, Pessolani MCV 1998. Mapping and identification of the major cell wall-associated components of Mycobacterium leprae. Infect Immun 66: 2525-2631.

Marques MAM, Espinosa BJ, da Silveira EKX, Pessolani MCV, Chapeaurouge A, Perales J, Dobos KM, Belisle JT, Spencer JS, Brennan PJ 2004. Continued proteomic analysis of Mycobacterium leprae subcellular fractions. Proteomics 4: 2942-2953.

Martins MVSB, da S Guimarães MM, Spencer JS, Hacker MAVB, Costa LS, Carvalho FM, Geluk A, van der Ploeg-van Schip JJ, Pontes MAA, Gonçalves HS, de Morais JP, Bandeira TJPG, Pessolani MCV, Brennan PJ, Pereira GMB 2012. Pathogen-specific epitopes as epidemiological tools for defining the magnitude of Mycobacterium leprae transmission in areas endemic for leprosy. PloS Neg Trop Dis 6: e1616.

Meima A, Smith WCS, van Oortmarssen GJ, Richardus JH, Habbema JDF 2004. The future incidence of leprosy: a scenario analysis. Bull WHO 82: 373-380.

Moet FJ, Pahan D, Oskam L, Richardus JH, the COLEP Study Group 2008. Effectiveness of single dose rifampicin in preventing leprosy in close contacts of patients with newly diagnosed leprosy: cluster randomized controlled trial. Brit Med J 336: 761-764.

Moet FJ, Pahan D, Schuring RP, Oskam L, Richardus JH 2006. Physical distance, genetic relationship, age and leprosy classification are independent risk factors for leprosy in contacts of patients with leprosy. J Inf Dis 193: 346-353.

Moet FJ, Schuring RP, Pahan D, Oskam L, Richardus JH 2008. The prevalence of previously undiagnosed leprosy in the general population of northwest Bangladesh. PLoS Negl Trop Dis 2: e198.

Oskam L, Slim E, Bührer-Sékula S 2003. Serology: recent developments, strengths, limitations and prospects: a state of the art overview. Lepr Rev 74: 196-205.

Reece ST, Ireton G, Mohamath R, Guderian J, Goto W, Gelber R, Groathouse N, Spencer J, Brennan P, Reed SG 2006. ML0405 ML2331 are antigens of Mycobacterium leprae with potential for diagnosis of leprosy. Clin Vacc Immunol 13: 333-340.

Ridley DS, Jopling WH 1966. Classification of leprosy according to immunity. A five-group system. Int J Lepr Other Mycobact Dis 34: 255-273.

Salgado CG, Ferreira DV, Frade MA, de S Guimarães L, da Silva MB, Barreto JG 2012. High anti-phenolic glycolipid-I IgM titers and hidden leprosy cases, Amazon Region. Emerg Infect Dis 18: 889-890.

Sampaio LH, Stephani MM, Oliveira RM, Sousa AL, Ireton GC, Reed SG, Duthie MS 2011. Immunologically reactive M. leprae antigens with relevance to diagnosis and vaccine development. BMC Infect Dis 11: 26.

Scollard DM, Adams LB, Gillis TP, Krahenbuhl JL, Truman RW, Williams DL 2006. The continuing challenges of leprosy. Clin Microbiol Rev 19: 338-381.

Spencer JS, Kim HJ, Marques MAM, Williams DL, Martins MVBS, Martins MLF, Lima MCBS, Sarno EN, Pereira GMB, Matos H, Fonseca LS, Sampaio EP, Ottenhoff THM, Geluk A, Cho S-N, Stoker NG, Cole ST, Brennan PJ, Pessolani MCV 2005. Identification of specific proteins and peptides in Mycobacterium leprae suitable for the selective diagnosis of leprosy. J Immunol 175: 7930-7938.

Spencer JS, Kim HJ, Wheat WH, Chatterjee D, Balagon MV, Cellona RV, Tan EV, Gelber R, Saunderson P, Duthie MS, Reece ST, Burman W, Belknap R, Mac Kenzie W, Geluk A, Oskam L, Dockrell HM, Brennan PJ 2011. Analysis of antibody responses to Mycobacterium leprae phenolic glycolipid-I, lipoarabinoman- 
nan and recombinant proteins to define disease-subtype specific antigenic profiles in leprosy. Clin Vacc Immunol 18: 260-267.

Torrelles JB, Khoo KH, Sieling PA, Modlin RL, Zhang N, Marques AM, Treumann A, Rithner CD, Brennan PJ, Chatterjee D 2004. Truncated structural variants of lipoarabinomannan in Mycobacterium leprae and an ethambutol-resistant strain of Mycobacterium tuberculosis. J Biol Chem 279: 41227-41239.

van Beers SM, Hatta M, Klatser PR 1999. Patient contact is the major determinant in incident leprosy: implications for future control. Int J Lepr Other Mycobact Dis 67: 119-128.
WHO - World Health Organization 2009. Enhanced global strategy for further reducing the disease burden due to leprosy: (plan period: 2011-2015), WHO/Regional Office for South-East Asia, New Delhi, 38 pp.

WHO - World Health Organization 2011. Leprosy update. Wkly Epidemiol Rec 36: 389-400.

Zhang J, Chatterjee D, Brennan PJ, Spencer JS, Liav A 2010. A modified synthesis and serological evaluation of neoglycoproteins containing the natural disaccharide of PGL-I from Mycobacterium leprae. Bioorg Med Chem Lett 20: 3250-3253. 